\title{
A Cluster-based Node Relocation Technique for Connectivity Restoration for Mobile Wireless Sensor Networks
}

Mahmood ul Hassan ( $\triangle$ mahmood.mscs@gmail.com )

Najran University https://orcid.org/0000-0002-8459-1471

Amin al-wady

Najran University

Khalid Mahmood

King Khalid University

Shahzad Ali

Al-Jouf University

Ibrahim Algamdi

Najran University

Muhammad Kashif Saeed

King Khalid University

Ansar Shah

Institute of Southern Punjab

\section{Research Article}

Keywords: Wireless sensor networks, transmission range, connectivity restoration, node failure, cluster

Posted Date: July 20th, 2021

DOl: https://doi.org/10.21203/rs.3.rs-526589/v1

License: (9) (i) This work is licensed under a Creative Commons Attribution 4.0 International License. Read Full License 


\title{
A Cluster-based Node Relocation Technique for Connectivity Restoration for Mobile Wireless Sensor Networks
}

\author{
Mahmood ul Hassan ${ }^{1}$, Amin al-wady $^{1,}$, Khalid Mahmood $^{2}$, Shahzad Ali ${ }^{3}$, Ibrahim Algamdi ${ }^{1}$, \\ Muhammad Kashif Saeed ${ }^{4}$, Ansar Munir Shah ${ }^{5}$ \\ ${ }^{1}$ Department of Computer Skills, Preparatory year, Najran University \\ Kingdom of Saudi Arabia. \\ ${ }^{2}$ Department of Information Systems, College of Science and Arts Mahayil Asir, King Khalid University, \\ Kingdom of Saudi Arabia. \\ ${ }^{3}$ Department of Computer Science, Jouf University \\ Kingdom of Saudi Arabia. \\ ${ }^{4}$ Department of Computer Science, Community College Mahayil Asir, King Khalid University \\ Kingdom of Saudi Arabia. \\ ${ }^{5}$ Department of Computer Science, Institute of Southern Punjab \\ Pakistan \\ * Correspondence: mahmood.mscs@gmail.com
}

\begin{abstract}
Wireless Sensor Networks (WSNs) are widely used in most applications of the Internet of Things (IoT) for efficiently sensing the harsh environments, collecting important data, and sending them to the base station for further analysis. Therefore, the research community worldwide is actively participating in addressing and solving the challenges associated with WSNs. The common issue that is being studied and well-thought-out are how to increase the network's life span by resolving node failure problem and efficient energy utilization. This paper introduces a Cluster-based Node Recovery (CNR) connectivity restoration mechanism based on the concept of clustering. The proposed technique utilizes a distributed cluster-based approach to identify the failed nodes, while Cluster Heads ( $\mathrm{CHs}$ ) play a significant role in the restoration of connectivity. The simulation results show that the proposed technique efficiently addresses node failure and restores the connectivity by moving fewer nodes than other existing connectivity restoration mechanisms.
\end{abstract}

Keywords: Wireless sensor networks; transmission range; connectivity restoration; node failure; cluster

\section{Introduction}

Wireless sensor networks are widely incorporated in many application areas to perform specific tasks. Advancements in wireless and sensor technologies have open many application areas in Wireless Sensor Networks (WSNs) such as battlefield surveillance, environment monitoring, healthcare, agriculture, and home automation [1]. Wireless sensor networks have gained tremendous attention from the industrial and research community. The rapid growth of WSNs is because it is composed of inexpensive tiny devices, having processing, sensing and, communication capabilities on a single integrated chip that can work on low-powered energy supply. As a result, many wireless sensor nodes are deployed to cope with sensing and computation tasks in WSNs. The area of interest for WSN is usually harsh or hostile, so the deployment of the wireless sensor nodes is without any pre-configured infrastructure. Thus, the connectivity and coverage restoration in WSN is a primary goal to be addressed.

The failure of sensor nodes partition the coverage area into disjoint segments, leaving multiple functional segments of sensor nodes inaccessible. The failed node in a WSN breaks the network's connectivity and causes the loss of important sensed information. The large scale node damage in WSN causes numerous disjoint segments in the network, and information from source sensor nodes to the base station could not be routed. Thus, the network's rapid connectivity restoration is significant for maintaining the network and observing and route the critical data to the remote centers. The deployment of additional sensor nodes is often impossible in 
harsh environments. Therefore, the process of connectivity restoration should be self-healing and self-organized with the efficient use of the existing sensor nodes.

Connectivity restoration plays a vital role during the operations of WSN. The tasks in WSN are distributed among all the sensor nodes. Due to the resource-constrained nature of WSN, it is essential to efficiently use the power, energy, and transmission ranges of the sensor nodes.

Clustering plays a significant role in achieving energy efficiency and scalability in WSNs. Nodes in a WSN can be combined in such a way that they form a hierarchical topology. This is achieved by making cluster heads, which are powerful nodes that manage many sensor nodes. Sensor nodes send their data readings to the respective cluster head, which performs data aggregation, eliminates the redundant data, and forwards it to the sink node. The cluster head may fail, which requires re-selection of the cluster head. Several approaches have been proposed in the literature to select the primary and backup cluster heads for each sensor node.

In this paper, we introduce a connectivity restoration mechanism based on the concept of clustering. The proposed technique is called Cluster-based Node Recovery (CNR). As connectivity restoration is based on clustering; therefore, it inherits all the pros associated with clustering. For achieving connectivity restoration, CNR moves a minimal number of nodes as compared to other existing techniques. Our approach utilizes a recovery mechanism called Wireless Broadcast Advantage to deal with one of the well-known cons of clustering known as disruption in inter-cluster communication. CNR uses a distributed cluster-based approach to identify the failed nodes, and for the restoration of connectivity, cluster heads play a significant role. By doing extensive simulation, we prove that CNR outperforms most of the existing state of the art approaches in multiple performance metrics.

The rest of the paper is organized as follows. In section 2, we elaborate on the literature review by explaining the most relevant related work associated with the considered problem. Section 3 presents the research method, while section 4 illustrates the results and analysis. The paper is concluded in section 5.

\section{Literature review}

The sensor field research started in the 1950s as the Sound Surveillance System (SOSUS) was the first sensor network to be introduced [1]. The research community has contributed to connectivity restoration a significant amount of work by considering multiple constraints that affect the connectivity restoration process in WSN. The primary focus of all the techniques designed so far for the connectivity restoration is node failure detection and recovery. The component or node failure is detected by failure detection, and it falls into single or collaborative diagnoses depending on the number of nodes that take part in the failure detection process.

The algorithm proposed in [2] uses a Markov Chain Controller to detect the faulty nodes through changes in the received aggregated path readings at the sink node. Sink nodes broadcast join messages in the network, and upon receiving this message, each node selects a suitable sink node by running any of the optimal path routing algorithms. During this process, there is a greater chance that the distribution of sensor nodes for each sink is not even; therefore, all sink nodes will collaborate to make the distribution even. Each sensor node will set its associated sink as the destination node and send a message containing its id, list of neighboring nodes, and IDs of nodes on route to the sink. After receiving all this information, each sink node will construct an aggregated path. Sink nodes will now set a time-period for their associated nodes to transmit the data. Each sensor node will send data readings frequently within the permissible time. After receiving data readings, the sink will construct a data table for each sensor node.

The technique presented in [3] deals with failure detection in cluster-based WSNs with the help of neighbor's coordination and cluster head detector. The selection of a cluster head depends on the node's residual energy. If the residual energy is greater than the precise threshold, the node becomes a cluster head. The process executes until all cluster heads are selected. After the cluster head selection process, each of the remaining nodes will have to join at least one cluster head. Cluster heads will now start receiving information from its member nodes. If information is received within a specific time-period, nodes will be considered normal, otherwise dead. The cluster head will now compute the packet delivery ratio for the nodes from which information is received and set an average value for the data packets delivery ratio. If the packet delivery ratio is greater than the average value, the node will be considered healthy; otherwise, it will be declared a faulty node.

The Fuzzy rule-based Faulty Node Classification and Management (FNCM) scheme proposed in [4] resolves the fault detection process by classifying the sensor nodes according to their hardware status. The cluster head detects the transmitter circuit fault, whereas the node itself detects the receiver circuit, sensor 
circuit, and battery fault. The fuzzy logic linguistic variables are used to diagnose and declare the hardware status of sensor nodes. The fuzzy logic rule-based system declares the nodes as normal, dead, traffic, and end node based on hardware status.

The research work done in PADRA [5] shows failure detection by one of the neighbors. The approaches like MPADRA [6] involve two neighbors, whereas the RIM [7] engages all the neighbors for failure detection.

The failure recovery process enables the network to perform its services at the pre-failure level or continues its services with graceful degradation. The recovery schemes proposed so far can be classified into single or cooperative, and it depends upon the participant nodes which take part in the recovery process. For example, the PADRA [5] uses a single elected neighbor to trigger and execute the recovery process whereas, the RIM [7] and C3R [9] offer all the neighbors to participate in the recovery process. Similarly, the work in DARA [8] and PADRA [5] offer two-hop information and cascaded relocation for recovery, which offers more overhead whereas, the RIM [7] and the C3R [9] only maintain 1-hop neighbor information. The model presented in [10] adopts various approaches like centralized and distributed by introducing mobile robots. A different approach involves three routing protocols that use the fact that different transmission ranges are possible that can prolong network lifetime is proposed in [11]. They use PEGASIS, LEACH, and VGA protocols.

F2CRA and P3CRA algorithms presented in [12] restore the connectivity by providing two vertex-disjoint and three vertex-disjoint paths between every pair of network nodes. The Connectivity Restoration with Assured Fault Tolerance (CRAFT) algorithm proposed in [13] is designed in such a way that it forms the Backbone Polygon (BP) surrounding the center of the partitioned network area. Relay Nodes (RNs) play a vital role in enhancing connectivity and coverage in an area of interest at a very low cost. RNs use two non-overlapping paths that connect each outer partition to the BP and ensure the connectivity restoration of the network.

In [18], the authors have proposed a novel connectivity restoration technique, namely "Intelligent On-Demand Connectivity Restoration for Wireless Sensor Networks (IDCRWSN)". IDCRWSN efficiently utilizes the residual energy and partial transmission range of the sensor nodes in an integrated manner to restore the network connectivity. The simulation results and analysis for IDCRWSN prove that the proposed technique is energy efficient and can better handle the node failure. Moreover, in the baseline approaches, large numbers of nodes participate in the connectivity restoration process resulting in more moved nodes. This was the major drawback of the baseline approaches, which is being addressed and solved by the IDCRWSN technique.

Efficient Solution for Connectivity Restoration (ESCR) [20] is an energy-efficient technique. The technique aims to restore the network with an efficient consumption of residual energy and least node movement. Only such node can participate in the network restoration, which is near to the faulty node and also have more energy.

In [21], the authors have proposed an energy efficient connectivity restoration technique, namely "Distributed Energy Efficient Node Relocation (DEENR)". It consumes less energy during the mobility of sensor nodes. In this technique no communication and mobility model is considered for the performance evaluation.

Keeping in view all the schemes mentioned above for node failure and connectivity restoration process in WSNs, it is observed that excessive processing is required to restore wireless sensor network connectivity. So to the best of our knowledge, CNR is the efficient technique that adopts a distributed cluster-based approach and imposes less overhead on the sensor nodes while detecting the node failure and restoring the connectivity.

\section{Research method}

\subsection{Problem formulation}

This research aims to propose a new technique for connectivity restoration that overcomes the shortcomings of the existing techniques. The following sets of assumptions are taken for the development of our proposed technique.

- A set of $\mathrm{N}$ sensor nodes are randomly deployed in a geographic region

- Sensor nodes are of two types. Cluster Heads (CHs) and ordinary sensor nodes.

- Cluster Heads are assumed to be powerful sensor nodes in terms of processing and battery

- Ordinary sensor nodes are sensor nodes that are less powerful than the CHs and have motion capabilities. 
- Cluster heads are responsible for identifying failed nodes (As the ordinary nodes send information periodically to $\mathrm{CHs}$; therefore, there is no need for any additional control messages).

\subsection{Energy model}

The proposed technique CNR transmits and receives a $\beta$-bit data packet over distance $d$ by utilizing the energy model described in [14]. While transmitting a $\beta$-bit data packet over distance $d$, the sensor node's energy depletion is calculated as:

$$
E_{T x}(\beta, d)=\left\{\begin{array}{ll}
\beta E_{\text {elec }}+\beta \varepsilon_{f s} d^{2} & d<d_{\circ} \\
\beta E_{\text {elec }}+\beta \varepsilon_{m p} d^{4} & d \geq d_{\circ}
\end{array}\right\}
$$

where $\varepsilon_{f s}$ is the required energy in free space by the Radio Frequency (RF) amplifier and $\varepsilon_{m p}$ is the required energy in multipath by the Radio Frequency (RF) amplifier. $E_{\text {elec }}$ is the energy depletion per bit for the sender or receiver circuit.

While receiving a $\beta$-bit data packet over distance $d$, the energy depletion of a sensor node is calculated as:

$$
E_{R x}(\beta)=E_{R x-e l e c} \beta
$$

where $E_{R x-e l e c}$ is the energy consumed per bit by the receiver circuitry.

The remaining residual energy of a sensor node is given by:

$$
E_{\text {reng }}(n)=E_{\text {max }}-E_{T x}(\beta, d)-E_{R x}(\beta)
$$

\subsection{System model}

It is considered that nodes are randomly deployed in an area of interest. All nodes have the same transmission range called $R_{c}$. It is assumed that all the nodes are divided into multiple clusters having a controlling cluster head for each cluster. The first step during the network operation is the selection of the cluster head. The cluster heads are selected based on the residual energy of the sensor nodes. For the details of the Cluster Head selection, the user is referred to one of our previous works [17]. Once the cluster heads are selected, then as the next step, the ordinary nodes start to send information to the cluster head. The cluster head will not only be responsible for the collection of sensed information from the ordinary nodes but also for the identification of the failed nodes. All the nodes associate with a single cluster head are referred to as its neighbors. Each ordinary node periodically sends the sensed information to the cluster head. After collecting information from all the neighboring nodes, the cluster head sends the accumulated information towards the sink in a multi-hop fashion. The formation of clusters and inter-cluster communication is illustrated in Fig 1. Clustering is an efficient approach in terms of energy consumption as it requires fewer messages to be exchanged among nodes, leading to a substantial increase in the network lifetime [12].

Clustering can prove to be an effective approach that can further be utilized to detect node failures in the network. Each cluster head contains all the neighbors' information like their location, their ID, their energy levels, etc. This information is exchanged by using the Hello messages. Each cluster head keeps track of the energy levels for the neighboring nodes. A threshold is defined, called $\mathrm{E}_{\mathrm{t}}$. If a cluster head does not receive a message from a neighboring node $\mathrm{N}_{\mathrm{i}}$, it checks to see if the $\mathrm{E}_{\mathrm{t}}\left(\mathrm{N}_{\mathrm{i}}\right)$ of that node during the previous exchange of the message was below the threshold. If both of these conditions are true, then it assumes that the node $\mathrm{Ni}$ has failed. As a next step, the cluster head decides whether the failed node's neighboring nodes need to be moved towards the failed node.

\subsection{Clustering-based Node Relocation (CNR)}

This section explains the working of our proposed protocol called Clustering-based Node Relocation (CNR). CNR makes effective use of clustering for the identification of failed nodes, as illustrated in the previous section. Once the cluster head detects a failed node, it has to make an important decision regarding the neighboring nodes' movement towards the failed node. The cluster head knows the location of each of the neighboring nodes, communication range $\left(\mathrm{R}_{\mathrm{c}}\right)$, and sensing Range $\left(\mathrm{R}_{\mathrm{s}}\right)$. The cluster head calculates the sensing area that will be affected $\left(A_{e}\right)$ by the failed node. $A_{e}$ can be seen as the intersection of the three sensing ranges of nodes $\mathrm{C}, \mathrm{D}$, and F, as shown in Fig 2. We define a threshold called At, which serves as a guideline for neighboring nodes' movement towards the failed node. $A_{t}$ is highly dependent upon the particular application or 
the situation. We assume $A_{t}$ to be 20 percent of the area of the cluster. If the $A_{e}$ exceeds the threshold value, then the cluster head calculates for each neighboring node of the failed node the distance $\mathrm{d} 1$ and $\mathrm{d} 2$ as shown in the figure. Excessive movement of each of the neighboring nodes towards the failed node may also decrease the overall sensing area. Therefore, it may not be beneficial for a specific neighbor to move it towards the failed node. The cluster head calculates the effective distance called $\mathrm{D}_{\text {effec }}$ to move each neighbor.

One interesting challenge that might occur regarding the restoration of connectivity is inter-cluster communication. As all the nodes in the network are mobile, there is always a possibility of disruption of connectivity among the cluster heads. To cope with this kind of disruption, we use a simple technique called Wireless Broadcast Advantage (WBA) [19]. The phenomenon is that when a wireless message is broadcasted, it is not only received by the receiver but also by the intermediate nodes that are present between the source and destination. Those nodes are called guard nodes. The figure below shows that nodes $\mathrm{C}$, F, and K (illustrated in blue color) are guard nodes. These nodes play an important part if the two cluster heads go out of each other's communication range. The idea is simple. When a cluster head sends an inter-cluster communication message towards the other cluster head, these guard nodes receive the message. They wait for an acknowledgment from the other cluster head. Absence of acknowledgment message means connectivity disruption between the two cluster heads. These guard nodes set a timer based on the residual energy such that the timer of a node having more energy level expires first and retransmits the inter-cluster message towards the destination cluster head. This technique mitigates the connectivity disruption among the cluster heads.

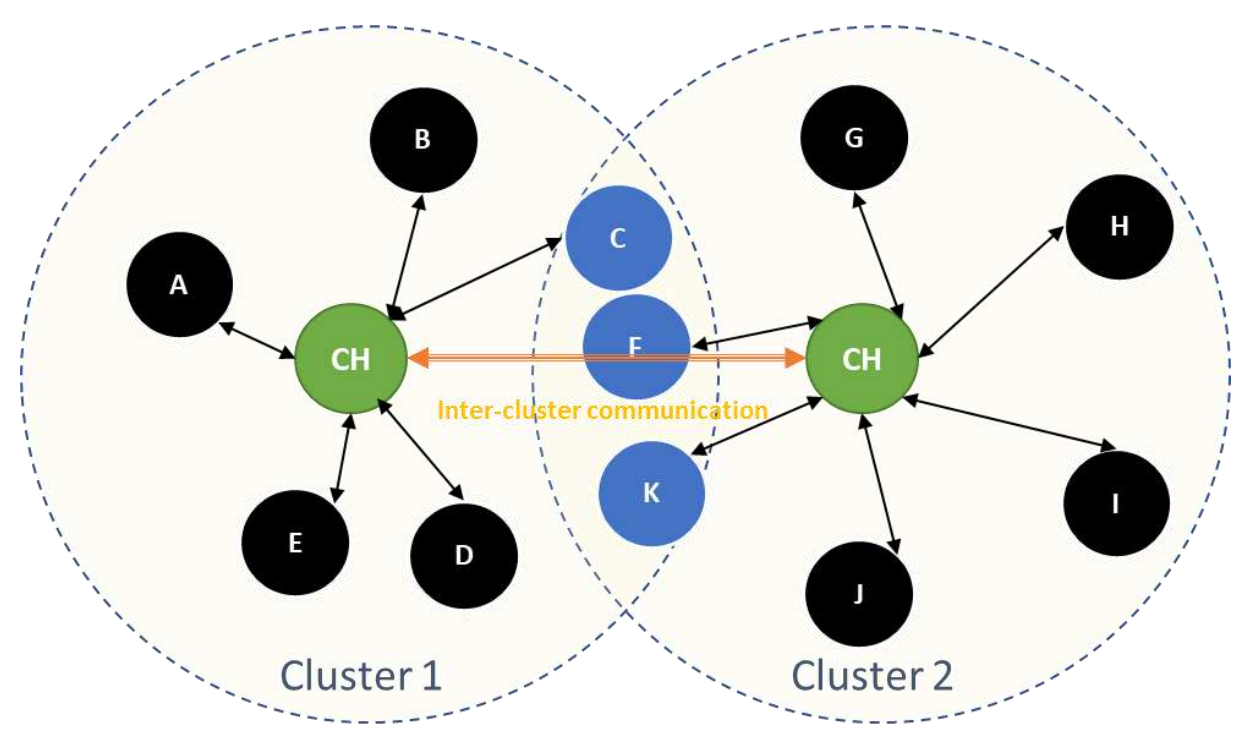

Figure 1: formation of clusters and inter-cluster communication 


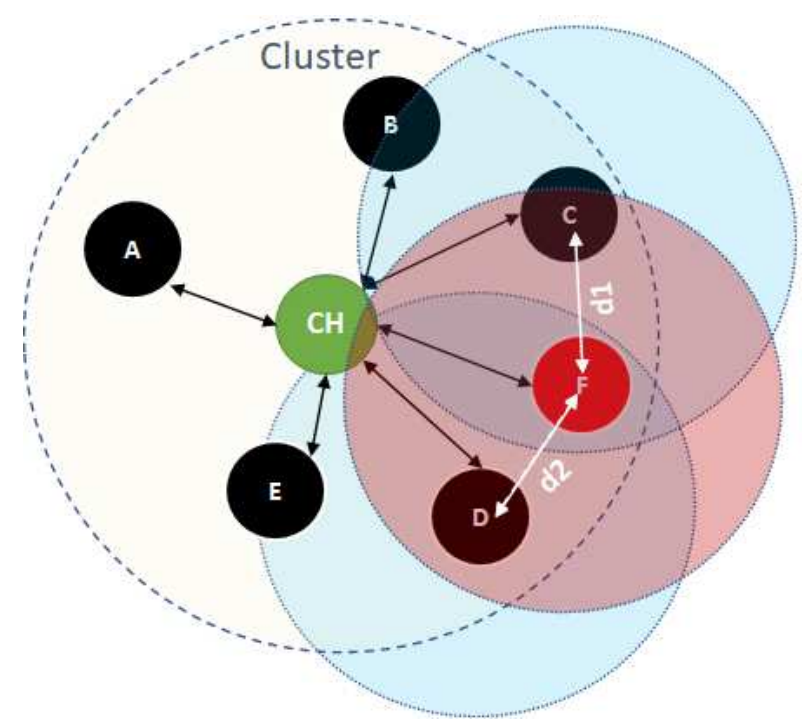

Figure 2: Detection of failed node and node relocation within a cluster

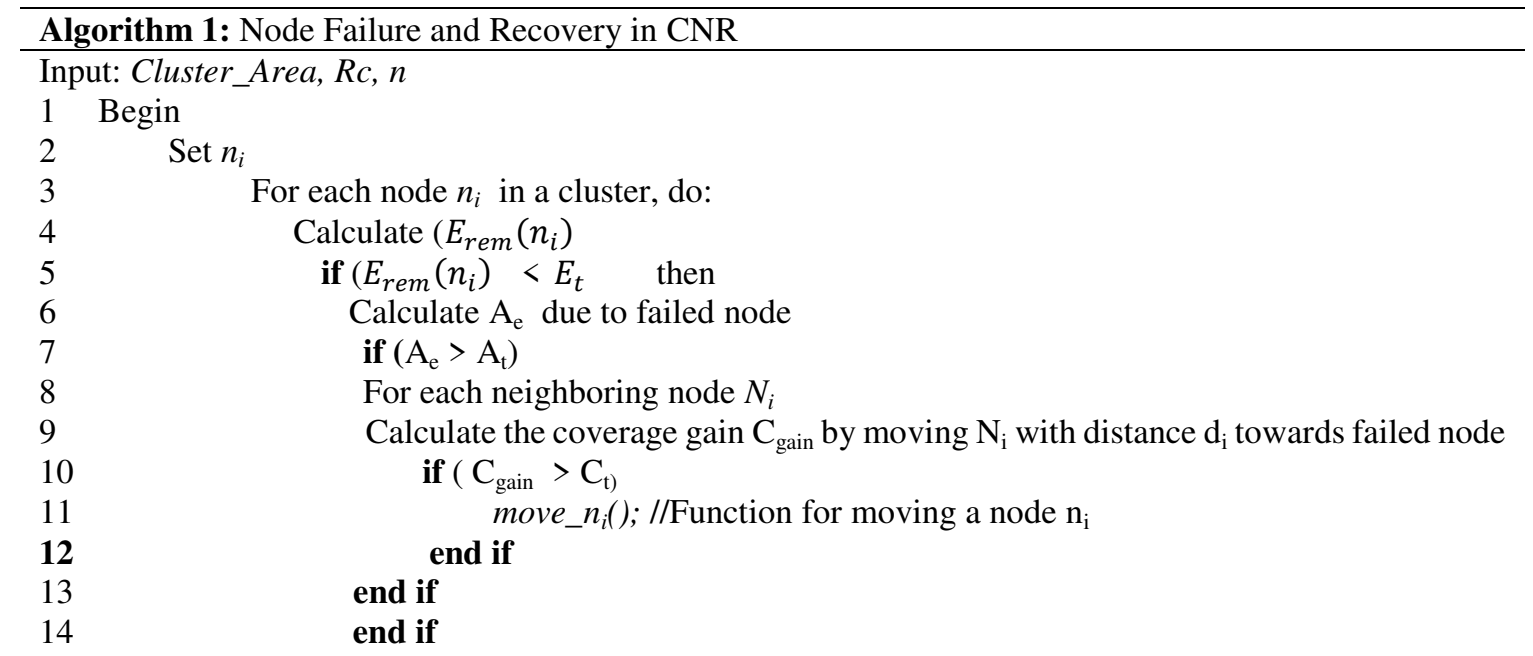

\section{Results and analysis}

To compare the proposed protocol with the existing baseline protocols, we utilized the INET framework [16] of the OMNeT++ simulator. The performance of the proposed protocol was compared with three existing techniques called $C^{3} R$ [9], VCR [15], and IDCRWSN [18]. The performance metrics used for comparison were the average number of nodes moved, total distance moved to restore connectivity, percentage coverage reduction, and the total number of exchanged packets. The simulation parameters are illustrated in Table 1 . Table 2 explains the objectives of the baseline algorithms. 
Table 1. Simulation parameters

\begin{tabular}{l|l}
\hline Parameters & Values \\
\hline Area & $800 \times 800 \mathrm{~m}^{2}$ \\
Number of nodes & $100-300$ \\
Sensing range & $15 \mathrm{~m}$ \\
Communication range & $250 \mathrm{~m}$ \\
The initial energy of sensor nodes & $25 \mathrm{~J}$ \\
$\mathrm{E}_{t}$ & $0.4 \mathrm{~J}$ \\
\hline
\end{tabular}

Table 2. Compared Baseline Techniques

\begin{tabular}{cccc}
\hline $\begin{array}{c}\text { Baseline } \\
\text { Techniques }\end{array}$ & Deployment of Nodes & $\begin{array}{c}\text { Participating nodes in the } \\
\text { connectivity restoration } \\
\text { process }\end{array}$ & Aim \\
\hline $\mathrm{C}^{3} \mathrm{R}$ & Random & All neighboring nodes & $\begin{array}{c}\text { Coverage-aware connectivity } \\
\text { restoration process }\end{array}$ \\
\hline $\mathrm{VCR}$ & Random & $\begin{array}{c}\text { Immediate neighbors based } \\
\text { on proximity }\end{array}$ & $\begin{array}{c}\text { Less number of nodes movement } \\
\text { for connectivity restoration }\end{array}$ \\
\hline IDCRWSN & Random & Only Care Taker nodes & $\begin{array}{c}\text { Connectivity restoration by moving } \\
\text { just the Care Taker nodes }\end{array}$ \\
\hline
\end{tabular}

\subsection{Number of nodes moved}

Figure 3 shows the performance of all the considered protocols in terms of the average number of nodes moved during connectivity restoration. It can be seen from the figure that among all the considered protocols, $\mathrm{C}^{3} \mathrm{R}$ results in the highest number of nodes moved. This phenomenon's major reason is cascaded relocation, where a large number of nodes need to move towards the failed node for the connectivity restoration process. Similarly, VCR also results in a more average number of nodes to be moved for the connectivity restoration process. Both VCR and $\mathrm{C}^{3} \mathrm{R}$ due to excessive movement of neighboring nodes for the connectivity restoration process results in a higher percentage reduction of field. IDCRWSN performs better than $C^{3} R$ and VCR because a special node called caretaker moves on-demand to restore the connectivity. Our proposed protocol CNR performs better as compared to the considered protocols. The benefit of using clustering for node relocation is evident from the figure. An increase in the number of nodes results in an increase in the average number of nodes moved for all the considered approaches. However, for CNR, we observe a decline in the average number of nodes moved as we increase the network's total number of nodes. The major reason behind this phenomenon is that as we increase the number of nodes in the network, the number of nodes in each cluster also increases. As the movement of nodes towards the failed node is controlled by the cluster head, and the decision is based on the reduction in the sensing area; therefore, more nodes in a cluster means moving a lesser number of nodes. 


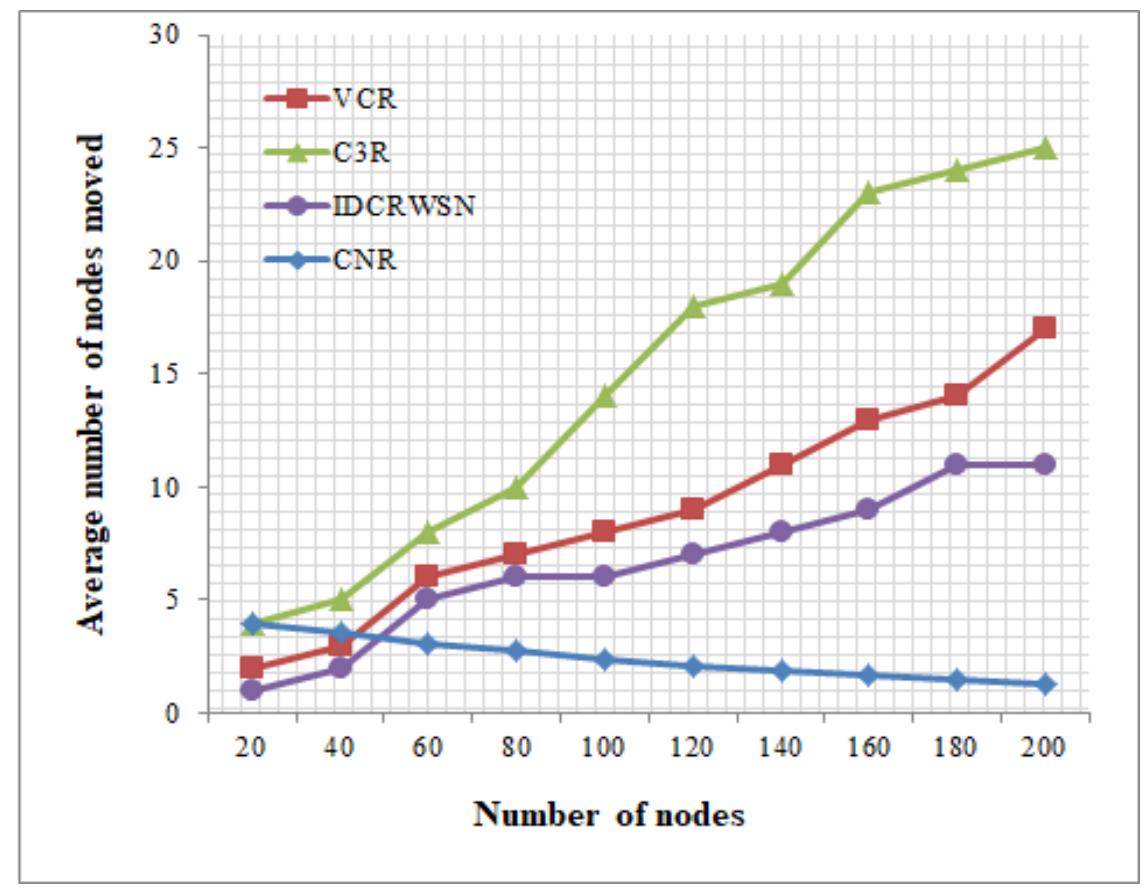

Figure 3. Average number of nodes moved in VCR, $C^{3} \mathrm{R}$ and IDCRWSN

\subsection{Coverage reduction}

Figure 4 illustrates the percentage reduction in field coverage for different communication ranges of the nodes. As the communication range increases, the field coverage reduction decreases for all the considered protocols. $\mathrm{C}^{3} \mathrm{R}$ and VCR perform worse as compared to the other considered protocols. The major reason is the cascaded relocation of excessive nodes, which causes the holes in coverage resulting in a drastic reduction in the field coverage. IDCRWSN is based on a centralized approach where the caretaker nodes are responsible for the connectivity restoration. The centralized control limits the coverage reduction more in IDCRWSN as compare to VCR and $\mathrm{C}^{3} \mathrm{R}$. Our proposed technique CNR outperforms all the baseline techniques in terms of reduction in field coverage. The percentage reduction in field coverage is minimal at the higher transmission ranges

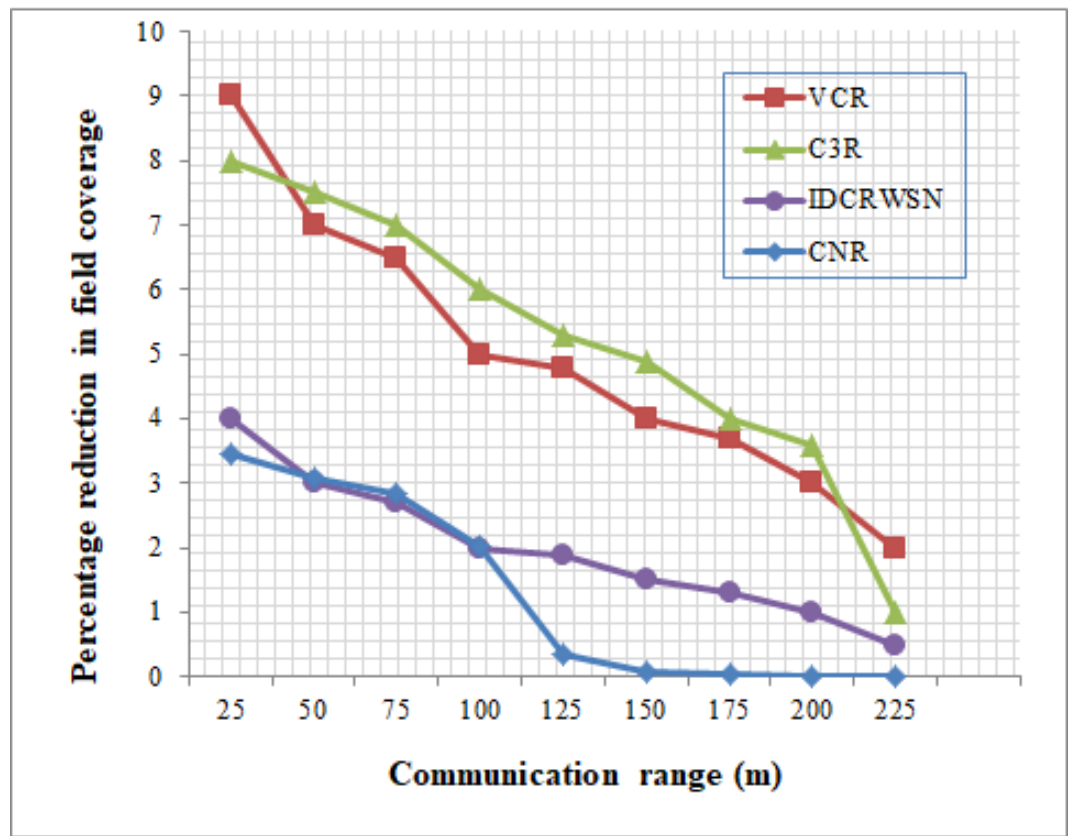

Figure 4. Percentage reduction in field coverage 


\subsection{Total distance moved during relocation}

The total distance moved by all the nodes in the network during connectivity restoration is illustrated in figure 5. The numbers of nodes in the network are varied between 20 to 200. It can be observed in figure 5 that as the number of nodes increases, the total distance moved during relocation increases for all the baseline protocols (VCR, C3R, and IDCRWSN). However, for our proposed protocol CNR, as the number of nodes in the network increases, the total distance moved during relocation decreases. This is due to the inherent characteristics of clustering. Clustering performs very well concerning scalability. Therefore, as the number of nodes in the network increases, the probability of finding a suitable neighbor close to the failed node also increases. Therefore, a lesser number of nodes need to be relocated for achieving the connectivity restoration. Moreover, CNR also moves just the minimal number of nodes for relocation and does not rely on cascaded relocation, which substantially reduces the total distance moved during relocation. Figure 5 shows two different variations of the CNR with $A_{t}$ equals to 10 percent and 20 percent, respectively. It can be observed from the figure that $A_{t}$ affects the performance. Reducing $A_{t}$ results in a slight increase in the total distance moved during relocation as reducing the threshold $\mathrm{A}_{t}$ results in moving more relocation nodes.

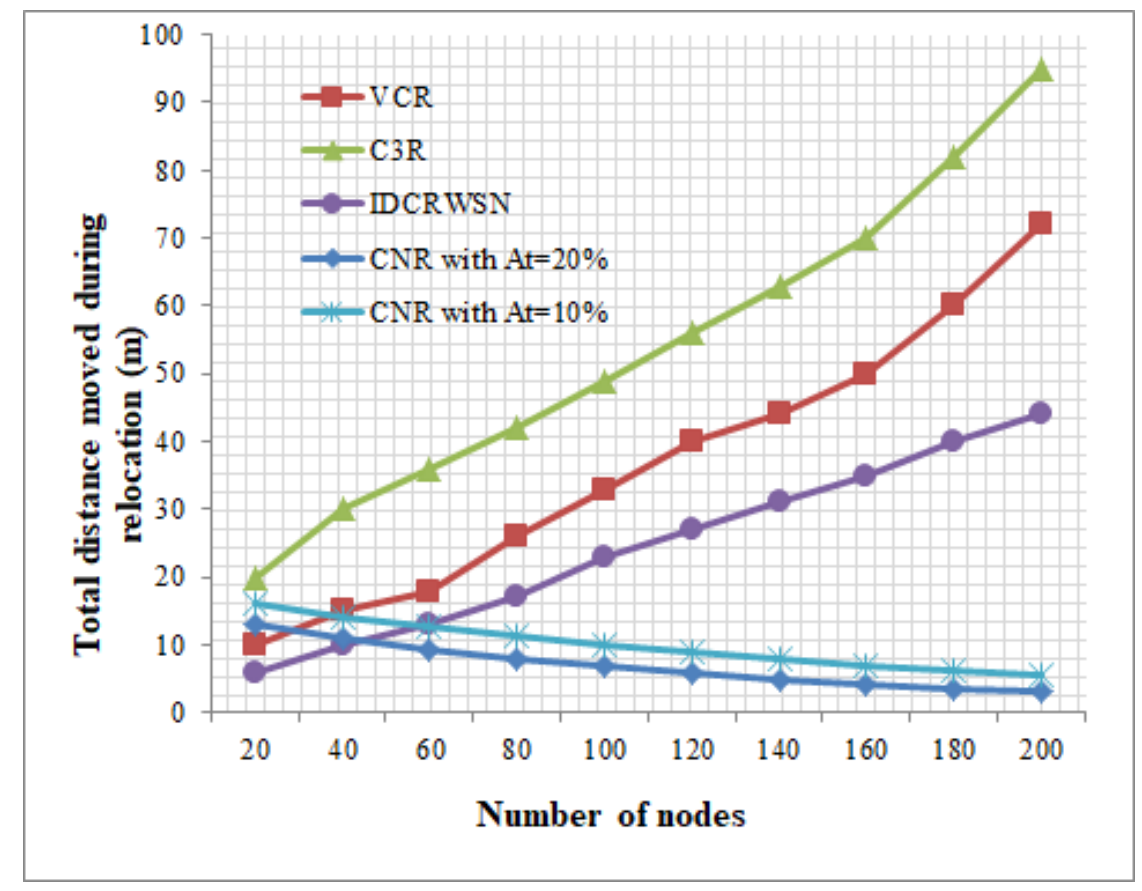

Figure 5. Nodes vs. distance moved

\subsection{Number of exchanged packets}

The proposed technique CNR exchanges a minimum number of packets among all the other baseline techniques. Clustering is known to reduce the number of transmitted packets and thus achieves energy efficiency. Moreover, figure 6 confirms the scalability aspect of the proposed technique. As the number of nodes increases, the total number of transmitted packets increases for all the baseline protocols. Our proposed technique CNR, results in the least number of packets transmitted. One major reason behind this is by utilizing clustering to reduce the number of control packets. Moreover, avoiding the excessive movement of the neighboring nodes of a failed node also results in the transmission of fewer packets. 


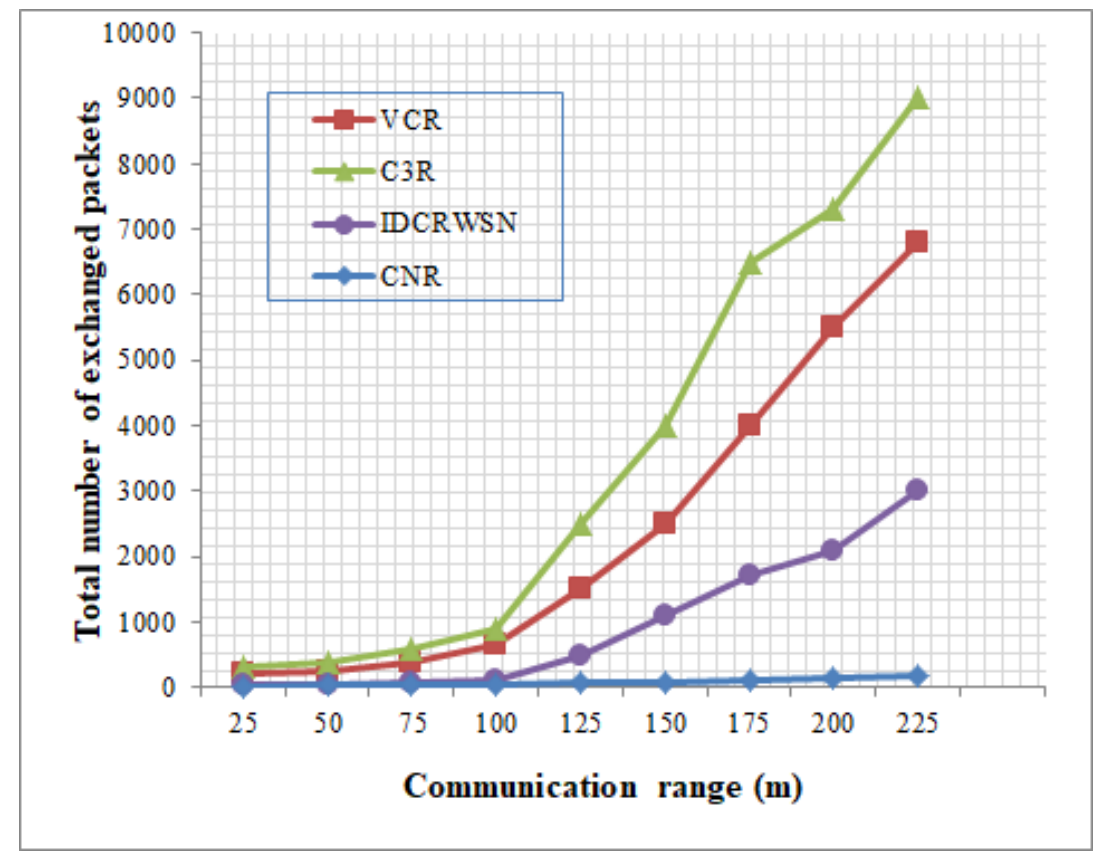

Figure 6. Total number of exchanged packets

\section{Conclusion}

In this paper, we proposed a novel connectivity restoration mechanism based on clustering. It moves a minimal number of nodes as compared to existing techniques for connectivity restoration. Moreover, it also utilizes a recovery mechanism called Wireless Broadcast Advantage for dealing with connectivity disruption during inter-cluster communication. Our approach utilizes a distributed cluster-based approach to identify the failed nodes, and for the restoration of connectivity, cluster heads play a very important role. In this work, we have compared our technique CNR with a variety of baseline techniques. The proposed approach takes full advantage of clustering and outperforms all the other considered protocols in terms of multiple performance metrics.

The future work can be extended in two possible dimensions. The first dimension involves developing an analytical model for the proposed solution under different mobility models. The second dimension involves implementation of the proposed solution on real sensor nodes and doing extensive performance evaluation.

\section{Acknowledgments:}

The authors extend their appreciation to the Deanship of Scientific Research at Najran University for funding this work through Research Groups under grant number (NU/ESCI/17/093 ).

Conflicts of interest: The authors declare that there is no conflict of interest. 


\section{References}

1. $\quad$ Akyildiz, Ian F., et al. "Wireless sensor networks: a survey." Computer networks 38.4 (2002): 393-422.

2. Artail, H., Ajami, A., Saouma, T., and Charaf, M. (2016) A faulty node detection scheme for wireless sensor networks that use data aggregation for transport. Wirel. Commun. Mob. Comput. doi:10.1002/wcm.2661.

3. Goyal, Rimpi, and Sukhwinder Singh Sran. "Fault Detection for the Cluster-Based System in Wireless Sensor Networks." Proceedings of the International Conference on Recent Cognizance in Wireless Communication \& Image Processing. Springer India, 2016.

4. Prasenjit Chanak, Indrajit Banerjee. 2016, Fuzzy rule-based faulty node classification and management scheme for large scale wireless sensor networks, Expert Systems with Applications Volume 45, 1 March 2016, Pages 307-321.

5. K. Akkaya, A. Thimmapuram, F. Senel and S. Uludag, "Distributed Recovery of Actor Failures in Wireless Sensor and Actor Networks," 2008 IEEE Wireless Communications and Networking Conference, Las Vegas, NV, 2008, pp. $2480-2485$.

6. K. Akkaya, F. Senel, A. Thimmapuram and S. Uludag, "Distributed Recovery from Network Partitioning in Movable Sensor/Actor Networks via Controlled Mobility," in IEEE Transactions on Computers, vol. 59, no. 2, pp. 258-271, Feb. 2010.

7. M. Younis, S. Lee, S. Gupta and K. Fisher, "A Localized Self-Healing Algorithm for Networks of Moveable Sensor Nodes," IEEE GLOBECOM 2008 - 2008 IEEE Global Telecommunications Conference, New Orleans, LO, 2008, pp. $1-5$.

8. Abbasi, AA, Akkaya, K, and Younis, M. 2007. A distributed connectivity restoration algorithm in wireless sensor and actor networks. International Proceedings of the 32nd IEEE conference on local computer networks (LCN 2007), Dublin, Ireland; October 2007.

9. Tamboli N, and Younis M. 2009. Coverage-Aware Connectivity Restoration in Mobile Sensor Networks. IEEE International Conference on Communications, ( ICC '09) Dresden, Germany. pp. 1-5.

10. Yongguo Mei, Changjiu Xian, Saumitra Das, Y. Charlie Hu and Yung-Hsiang Lu. 2006. Replacing Failed Sensor Nodes by Mobile Robots. Proceedings of the 26th IEEE International Conference on Distributed Computing Systems Workshops (ICDCSW'06).

11. Amer A. Al-Rahayfeh, Muder M. Almi'ani, and Abdelshakour A. Abuzneid. 2010. Parameterized Effect Of Transmission Range On Lost Of Network Connectivity (LNC) Of Wireless Sensor Networks. International Journal of Wireless \& Mobile Networks (IJWMN), Vol.2, No.3, August 2010.

12. Yali Zeng, $\mathrm{Li} \mathrm{Xu}$ and Zhide Chen, 2016. Fault-Tolerant Algorithms for Connectivity Restoration in Wireless Sensor Networks Sensors 2016, 16, 3; doi: 10.3390/s16010003.

13. S. Lee et al., Connectivity restoration in a partitioned wireless sensor network with assured fault tolerance, Ad Hoc Netw. (2014), http://dx.doi.org/10.1016/j.adhoc.2014.07.012.

14. Chanak, Prasenjit, Indrajit Banerjee, and R. Simon Sherratt. "Energy-aware distributed routing algorithm to tolerate network failure in wireless sensor networks." Ad Hoc Networks56 (2017): 158-172.

15. M. Imran, M. Younis, A. Md Said and H. Hasbullah, "Volunteer-instigated connectivity restoration algorithm for Wireless Sensor and Actor Networks," 2010 IEEE International Conference on Wireless Communications, Networking and Information Security, Beijing, China, 2010, pp. 679-683. doi: 10.1109/WCINS.2010.5544679

16. Varga, András, and Rudolf Hornig. "An overview of the OMNeT++ simulation environment." Proceedings of the 1st international conference on Simulation tools and techniques for communications, networks and systems \& workshops. ICST (Institute for Computer Sciences, Social-Informatics and Telecommunications Engineering), 2008.

17. Ali Shahzad, Madani, Sajjad. "Distributed efficient multihop clustering protocol for mobile sensor networks". The International Arab Journal of Information Technology 2011; 8(3): 302- 309.

18. Mahmood, K., Khan, M.A., Shah, A.M., Ali, S. and Saeed, M.K. "Intelligent On-Demand Connectivity Restoration for Wireless Sensor Networks". Wireless Communications and Mobile Computing, 2018.

19. Huang X., Zhai H. and Fang Y., "Lightweight Robust Routing in Mobile Wireless Sensor Networks", Military Communications Conference, 2006 MILCOM 2006, Page(s):1 - 6, 23-25 Oct. 2006.

20. Saeed, M.K., ul Hassan, M., Mahmood, K. et al. Efficient Solution for Connectivity Restoration (ESCR) in Wireless Sensor and Actor-Networks. Wireless Pers Commun (2020). https://doi.org/10.1007/s11277-020-07962-3

21. M. Hassan, M. Amir, S. Ali, K. Mahmood and A. Munir, "Distributed Energy Efficient Node Relocation Algorithm (DEENR)", International Journal of Advanced Computer Science and Applications, vol. 9, no. 3, 2018. Available: 10.14569/ijacsa.2018.090315 